\title{
Knowledge assessment of Sepsis Diagnosis and Management during COVID-19 pandemic among House Officers \& Medical Officers in Ibrahim Malik Teaching Hospital in Khartoum State, Sudan, 2021
}

\section{ElMuiz Abdelrahman}

Medical student, University of Khartoum, Faculty of Medicine, Khartoum, Sudan

Khabab Abbasher Hussien Mohamed Ahmed

Medical student, University of Khartoum, Faculty of Medicine, Khartoum, Sudan

Ibrahim Mahgoub

Medical Student, University of Khartoum, Faculty of Medicine, Khartoum, Sudan

Mohammed Eltahier Abdalla Omer ( $\square$ Mohammedeltahier100@gmail.com )

MBBS, Gadarif University Faculty of Medicine and Health Sciences

Yassin Abdelrahim Abdalla

MBBS, Omdurman Islamic University, Faculty of Medicine and Health Sciences

Abdelgadir Ali Bashir

Associate professor of Community Medicine, University of Khartoum, Faculty of Medicine, Khartoum,

Sudan

Research Article

Keywords: Knowledge, Sepsis, COVID-19, House Officers, medical officer

Posted Date: June 2nd, 2021

DOI: https://doi.org/10.21203/rs.3.rs-577986/v1

License: (c) (i) This work is licensed under a Creative Commons Attribution 4.0 International License.

Read Full License 


\section{Abstract}

Background: Sepsis is life-threatening and frequently a final common pathway to death for many infectious diseases worldwide and may lead to death if left untreated. The objective is to assess the scientific medical knowledge upon sepsis among House officers and Medical officers in terms of diagnosis and management.

Method: An observational cross sectional Hospital-based study was conducted through self-administered retrospective questionnaires based on the objective of the study. We enrolled 155 participants through Convenience sampling. The questionnaire contains parts of validated Sepsis criteria in terms of diagnosis and management. Analysis was done by using SPSS version 20. Data are presenter as frequencies and percentages using figures and tables. $P$ value less than 0.05 considered significant Mann Whitney $U$ test use to compare level of knowledge adherence between trained participants and nontrained participants.

Results: The mean knowledge score about sepsis diagnosis was 2.6 out of $10(\mathrm{SD}=1.8)$, and about sepsis management was 2.8 out of $8(S D=1.8)$ the mean overall score was 5.5 out of 18 .

No significant difference found between participants who were trained upon diagnosis and management of sepsis and those who were not.

Conclusion: Study findings illustrated that the capacity to perceive and manage sepsis among House Officers and Medical Officers doctors is poor and there are dangerous gaps in their investigation and management of such septic patients.

\section{Introduction}

\subsection{Background}

Sepsis is a life-threatening infection syndrome reaction, which is usually the common end of death for many infectious diseases around the world.. It involves organ dysfunction caused by a dysregulated host response to infection and may lead to death if left untreated. [1] Sepsis also represents a global health problem in terms of morbidity, mortality, social and economic costs. Although usually managed in Intensive Care Units, sepsis showed an increase prevalence among Internal Medicine wards in the last decade. This is substantially due to ageing of population and to multi-morbidity. [3] However, there is consensus that the incidence is increasing, driven by an ageing population with multiple comorbidities, increased use of immunosuppressive therapy and high-risk interventions. Mortality estimates for sepsis range from $27 \%$ to 36 ; however, the risk of death from sepsis has been falling over the recent decades despite the increasing incidence, perhaps due to improvement in care. [6] In the community, sepsis is usually a clinical exacerbation of a common and preventable infection. Sepsis also frequently results from infections acquired in health care settings, which are one of the most frequent adverse events during care delivery and affects hundreds of millions of patients worldwide every year. [1] Recent 
progress in sepsis research has been able to improve the knowledge about the basic pathophysiological process of sepsis. However, in daily ICU practice it remains difficult to identify and treat sepsis, its related conditions, adequately. There are still concerns about the lack of a consistent definition and understanding of sepsis in the global medical community. [7] Sepsis is the presence of a documented or strongly suspected infection, with a systemic inflammatory response, as indicated by the presence of some of the following features- body temperature greater than $38^{\circ} \mathrm{C}$ or less than $36^{\circ} \mathrm{C}$, heart rate greater than 90 beats/min, respiratory rate greater than 20 breaths/min or hyperventilation with $\mathrm{PaCO} 2$ less than $32 \mathrm{mmHg}$, White blood cell count $>12,000 / \mathrm{mm}^{3},<4,000 / \mathrm{mm}^{3}$, or with $>10 \%$ immature neutrophils. Severe sepsis is sepsis complicated by acute circulatory failure characterized by persistent arterial hypotension, despite adequate volume resuscitation, and unexplained by other causes. [7]

In the case of COVID-19, the effects on the respiratory tract are known, and most people are hospitalized and develop pneumonia to varying degrees; however, virtually all other organ systems are affected. When available, the Global Sepsis Alliance can more clearly emphasize that COVID-19 does cause sepsis. Approximately 2-5\% of COVID-19 patients show typical signs of multiple lesions of sepsis after approximately 8-10 days. COVID-19 died of sepsis and its complications, so it is important to understand and recognize the early signs of sepsis and start treatment immediately after the diagnosis is made. [13]

The Global Sepsis Alliance stated that COVID-19 can cause sepsis, which is the body's overwhelming lifethreatening response to infection, which can lead to organ damage or death.[14]

Sepsis is a life-threatening syndromic response that my lead to death if left untreated. Sepsis often presents as the clinical deterioration of common and preventable infections. The increase in the incidence and severity of sepsis may be related to population aging, immunosuppression and multidrug resistant infections. On this connection, the majority of cases of sepsis (about 60-80\%) have been observed in patients over 65 years old, with a 30-times increase in over 85 years old. At present, most of patients referring to Emergency Department (ED) share the above-mentioned features (Eg old age, chronic diseases) and quite a lot of sepsis patients are hospitalized from emergency room to non-intensive care unit.[3] Since diseases caused by infections are common in Sudan thus Sepsis cases will always be present. However, sepsis being a preventable case if diagnoses early and managed adequately. Thus House Officers and Medical Officers must have sufficient knowledge regarding the diagnosis, management and other important medical information regarding sepsis otherwise the cases will be missed on its early stages or not managed sufficiently or any form of low performance which will cause the septic patient to die a preventable death. Sepsis remains a major cause of morbidity and mortality worldwide, with increased burden in low- and middle- resource settings.

Sepsis and COVID-19 have many similarities, but the direct shift of sepsis management to COVID-19 management requires a little care.The pathophysiology of COVID-19 is currently not fully understood and seems to vary from person to person. Sepsis remains a major health burden worldwide and all protocols are poor. It is also important to note that further evaluation is needed in the context of COVID-19. In fact, 
with fast collaborative efforts towards COVID-19, perhaps sepsis management can ultimately see more benefits from COVID-19. [15]

Our research has assessed both the House officers and Medical officers' knowledge upon diagnosing and properly managing sepsis cases in Ibrahim Malik Teaching hospital. Thus my findings are of substantial value because it reflects whether the House officers and Medical officers in Ibrahim Malik Teaching hospital are will prepared to deal with life threatening cases of Sepsis and potentially saving their patients' lives. Also my findings are determining whether further trainings should be conducted regarding sepsis diagnosis and management thus not to let septic patients die a preventable death. It is shedding the light to Medical colleges and Hospital medical staff to give this topic more attention for the future and current House and Medical officers.

\subsection{OBJECTIVES}

\section{General Objectives:}

1. To Asses House Officers and Medical Officers knowledge upon diagnosis and management of Sepsis in Ibrahim Malik Teaching Hospital in 2021.

\section{Specific Objectives:}

1. To assess House/Medical officers' knowledge upon diagnosis, management of Sepsis as well as well as SOFA (Sequential Organ Failure Assessment)

2. To assess House/Medical officers' basic knowledge about SIRS (Systemic inflammatory response syndrome)

3. To assess the impact of previous clinical experience between House officers and Medical officers on their knowledge performance.

\section{Material And Methods}

\section{- Study Design:}

This research is a descriptive cross-sectional Hospital-based study.

\section{- Study Area:}

Ibrahim Malik Teaching Hospital. The hospital is in Alsahafa east, Khartoum city, Khartoum state, Sudan. It covers Khartoum state mainly. It is a public hospital. It contains COVID-19 isolation ward. Regarding the working staff, there are 200 house officers, 50 medical officers, 10 Obs \& Gyne specialists, 4 pediatric specialists, 8 medicine specialists. 9 surgery specialists, 2 physiotherapists, 2 radiologists and 7 Obs \& Gyne consultants. 7 pediatric consultants, 9 medicine consultants, 7 surgery consultants. 226 Nurses and sisters work there. Regarding the units there are 10 units: Radiology unit, Obstetrics and Gynecology unit, 
Pediatric unit, 8 Medicine units, 10 Surgery units, Accident and emergency unit, central care unit, Nursing unit, Physiotherapy unit and Central lab. The number of beds in the hospital is 439 beds.

\section{- Study Population:}

House officers and Medical officers of Ibrahim Malik Teaching Hospital.

\section{- Sampling Technique:}

The technique used for sampling is Convenience sampling.

\section{- Sample Size:}

- The sample size is calculated by the formula

$$
n=\frac{Z^{2} p q}{d^{2}}
$$

The Sample size $(n)=192$.

Where:

$\mathrm{n}$ : The sample size

Z: The degree of confidence which equals $1.96 \mathrm{p}$ : The prevalence.

$q:$ is $1-p$

d: The margin of error.

\section{- Sample Unit:}

House officers and Medical officers.

\section{- Tools and Date collections:}

The data was collected using interview-based questionnaires. This questionnaire is an author-structured questionnaire with the objective of assessing the knowledge upon Sepsis diagnosis and management. The criteria used in this questionnaire was inspired from a paper done by Keeley A et al. [12] The questionnaire contains four sections. Section One contains written consent \& Socio-demographic informations. Section Two involves General knowledge and Insight about sepsis, including assessing the impact on performance on previously trained Officers upon sepsis. As well as assessing their insight on their knowledge upon sepsis diagnosis and management. Section Three deals with Sepsis diagnosis 
criteria, the criteria included SIRS criteria, Severe sepsis criteria, Septic shock criteria and finally qSOFA score criteria. Section Four composed of Sepsis treatment and management.

- Sepsis six management bundle was used as the assessment base.

\section{- Inclusion Criteria:}

All House officers and Medical officers in Ibrahim Malik Teaching hospital.

\section{- Exclusion Criteria:}

Anyone who refuses to fill in the questionnaire

As well any health worker other than House officer and Medical officer working in Ibrahim Malik

\section{- Data Analysis:}

Analysis was done by using SPSS version 20. Data are presenter as frequencies and percentages using figures and tables. $P$ value less than 0.05 considered significant. Mann Whitney $U$ test use to compare level of knowledge adherence between trained participants and non-trained participants.

\section{Results}

\section{Baseline characteristics:}

We enrolled 155 participants $66 \%$ of them was female, mean age was $25(S D=1.7) 66 \%$ was house officer and $55 \%$ receive training upon how to diagnose, manage and prevent sepsis. (Table 1 )

\section{Table 1: Baseline characteristics}




\begin{tabular}{|lll|}
\hline Baseline characteristics & & Frequencies (\%) \\
\hline Gender & Female & $103(66.5 \%)$ \\
\hline Occupation & Male & $52(33.5 \%)$ \\
\hline Clinical experience & House officer & $103(66.5 \%)$ \\
\hline & Medical officer & $52(33.5 \%)$ \\
\hline & One year & $43(27.7 \%)$ \\
\hline Training in sepsis & Two year & $20(12.9 \%)$ \\
\hline & $>$ Two year & $5(3.2 \%)$ \\
\hline & No & $69(44.5 \%)$ \\
\hline
\end{tabular}

\section{Insight about sepsis:}

Majority of participants stated that they are not too knowledgeable about sepsis, its treatment and complications associated with it (62\%). $57 \%$ view sepsis as a condition and $21 \%$ view it as a syndrome $61 \%$ stated that they know difference between sepsis and septic shock. (Figure 1, 2 and 3 )

\section{Knowledge about sepsis diagnosis:}

On asking 10 questions related to knowledge about sepsis diagnosis the most answered wrongly question was the platelet count on sepsis ( $92 \%$ answered wrongly) followed by creatinine and bilirubin level ( $88 \%$ answered wrongly). the most answered correctly question was leukocytes status (55\% answered correctly). (figure 4) $30 \%$ of participants know that the only difference between systemic inflammatory response syndrome (SIRS) and sepsis is presence of infection. (Figure 5) On asking about qSOFA criteria $19 \%$ know that respiratory rate must be more than 22 breaths per second, $16 \%$ know that systolic blood pressure must be more than $100 \mathrm{mmHg}$ and $29 \%$ knows that Glasgow coma scale is included specifically in qSOFA criteria. (Figure 6, 7 and 8)

\section{Knowledge about sepsis treatment and management:}

On asking eight question regarding sepsis management majority of participants did not know what meant by sepsis six bundle, the most answered wrongly question was glucose level target ( $88 \%$ answered wrongly) the most answered correctly question was type of antibiotic used (65\% answer correctly). (Figure 9) 


\section{Knowledge Score:}

The mean knowledge score about sepsis diagnosis was 2.6 out of $10(\mathrm{SD}=1.8)$, and about sepsis management was 2.8 out of $8(S D=1.8)$ the mean overall score was 5.5 out of 18 . (Table 2$)$ No significant difference found between participants who were trained upon diagnosis and management of sepsis and those who were not. (Table 3)

Table 2: Knowledge level of participants

\begin{tabular}{|lllll|}
\hline & $\begin{array}{l}\text { Mean } \\
(\text { SD) }\end{array}$ & Minimum & Maximum & $\begin{array}{l}\text { Participant } \\
\text { who score } \\
\geq 50 \%\end{array}$ \\
\hline $\begin{array}{l}\text { Knowledge level about sepsis diagnostic } \\
\text { criteria (out of 10) }\end{array}$ & $\begin{array}{l}2.6 \\
(1.8)\end{array}$ & 0 & 8 & $18 \%$ \\
\hline $\begin{array}{l}\text { Knowledge level about sepsis management } \\
\text { criteria (out of 8) }\end{array}$ & $\begin{array}{l}2.8 \\
(1.8)\end{array}$ & 0 & 7 & $37 \%$ \\
\hline Overall (out of 18) & $\begin{array}{l}5.5 \\
(3.2)\end{array}$ & 0 & 13 & $19 \%$ \\
\hline
\end{tabular}

Table 3: Knowledge level difference between trained and non-trained participants

\begin{tabular}{|lcccc|}
\hline & Trained & Not trained & P value \\
\hline Median knowledge level about sepsis diagnostic criteria & 3 & 2 & 0.2 \\
\hline Median knowledge level about sepsis management criteria & 3 & 3 & 0.19 \\
\hline Median Overall knowledge & 6 & 5 & 0.17 \\
\hline
\end{tabular}

\section{Discussion}

It is clear that timely diagnosis and management of sepsis saves lives and reduces morbidity. This relies on prompt recognition and definitive management by the healthcare team. Here we show that junior doctors (House officers and Medical officers) are not able to recall the key SIRS criteria that enable recognition of sepsis. This study investigated the knowledge of Sepsis diagnosis and management among House officers and Medical officers in Ibrahim Malik teaching Hospital in Khartoum state 2021. We enrolled 155 participants $66 \%$ of them was female, mean age was 25 (SD=1.7) $66 \%$ was house officer and $55 \%$ receive training upon how to diagnose, manage and prevent sepsis. My Study showed that the 
knowledge House Officers and Medical officers in Ibrahim Malik teaching Hospital regarding Sepsis results were poor whereas only $18 \%$ of the participants score below $50 \%$ of Sepsis and SIRS diagnostic criteria in similar to a study done by Sarah L Cowan, Jonathon AA Holland, lan Frost, Andrew D Kane, in Jun 2016 in the Royale college of Physicians, through a questionnaire designed by the authors (Sepsis questionnaire, S1) was distributed to all foundation year 1 (FY1) doctors and senior house officers (SHOs; a mixture of foundation year two and CT1 core medical trainees) attending a weekly teaching session during a district general hospital. Doctors were from a spread of various specialties. Results showed only $4 \%$ of respondents correctly listed all of the SIRS criteria. $50 \%$ of junior doctors may a minimum of partly outline sepsis. [8]. The poor knowledge of sepsis diagnostic criteria could be due to global problem of accurate definition of sepsis but most likely the reason in my study is the poor training in both College and hospital setting. Regarding sepsis management my study showed that the majority of participants did not know what meant by sepsis six bundle in contrast to Junior doctors appeared to be more familiar with the Sepsis Six care bundle [8]. This difference in familiarity with Sepsis Six bundle is most likely due to poor training in both college and hospital settings. My study showed that only $17 \%$ of the participants answered correctly the minimum I.V. volume to be administered in similar to a study which showed that there were 15/231 (6.5 per cent) correct responses. a study done by Michael Courtney, Bussa Gopinath, Matthew Toward, Rajesh Jain, Milind Rao done in in two UK hospitals, with 77 questionnaires were completed. In 2014 Participants were asked the fluid volume challenge that they would prescribe for each patient. Responses were compared with the Surviving Sepsis Campaign's recommended volume during the study $(20 \mathrm{ml} / \mathrm{kg})$. [9]. This similarity in poor management results only shows that its due to poor training and focus upon sepsis as a topic regarding its management. Even though $55 \%$ of the participants received training upon Sepsis, the results showed no significant difference between the trained and the non-trained nor the clinical experience between House officers and Medical officers whereas the overall median score knowledge to the trained participants is 6 in similar to the score of 5 to the non-trained participants in similar to a study done by Michael Courtney, Bussa Gopinath, Matthew Toward, Rajesh Jain, Milind Rao done in in two UK hospitals, with 77 questionnaires were completed. In 2014 that showed there was no significant difference between doctor grades (FY1 and $\mathrm{SHO}$ ) in any scenario [9]. This is most likely that Sepsis as topic overall is complicated when it comes to definition and perception of its diagnostic criteria, and poor training might have a significant role in it as well. In my study assessments of participants' insight about their knowledge it showed that the majority of participants stated that they are not too knowledgeable about sepsis, its treatment and complications associated with it (62\%) in contrast to a study was done by Elizabeth Roebuck in Sept. 2015 in North England with over 144 clinicians completed the survey, gaining a $21.8 \%$ response rate. $54 \%$ of clinicians felt like they had good knowledge, leaving $46 \%$ of clinicians feeling a lack of knowledge [10]. This difference in knowledge and confidence is most likely attributed to the quality of training in college level and the hospital setting. In my study asking about qSOFA criteria $19 \%$ know that respiratory rate must be more than 22 breaths per second, 16\% know that systolic blood pressure must be more than $100 \mathrm{mmHg}$ and $29 \%$ knows that Glasgow coma scale is included specifically in qSOFA criteria. Thus overall poor performance in similar to a study done by Merijn C F Mulders, Feike J Loots, Joey van Nieuwenhoven, Jan C ter Maaten, Hjalmar R Bouma in March 2021 Two thousand five hundred and sixty GPs were 
invited and 229 agreed to participate in a survey, reached out to through e-mail and WhatsApp groups. And mainly the results showed that few of the responding GPs had heard of the qSOFA (27.7\%) [11]. This clear lack of knowledge of qSOFA criteria and its importance in early septic patients' assessment is most likely manifested in poor training in both college and hospital settings.In my study regarding the participants' knowledge upon the main difference between SIRS and Sepsis is that $30 \%$ of participants know that the only difference between systemic inflammatory response syndrome (SIRS) and sepsis is presence of infection.

\section{Conclusion}

The Study findings illustrated that the capacity to perceive and manage sepsis among House Officers and Medical Officers doctors in Ibrahim Malik teaching Hospital is poor and there are dangerous gaps in their investigation and management of such septic patients.

\section{RECOMMENDATION}

All House Officers and Medical officers included in ward cover or Emergency admission should be given not only by delivering further teaching on sepsis to junior doctors in our hospitals. but moreover an aidememoire to encourage the convenient acknowledgment and treatment of this common and unsafe syndrome. In regards to undergraduates my recommendation for the college settings is to provide sufficient curriculum for Sepsis diagnosis and management in as main life threatening complication in the field of Microbiology.

\section{LIMITATIONS}

Firstly, the practical difficulties in the application of prospective approaches, given the busy, and erratic schedules of House Officers and Medical officers. Secondly, during my data collection all the House officers went on a strike which made physical data collection not possible. Thus virtual collection through (Google forms) was the only way and the response rate was poor due to lack of cooperation with my targeted group as well as Telegram banning my account due to Spam alert in reasoning of sending to too many strangers accounts. Finally, the number of House officers and Medical officers in Ibrahim Malik Teaching hospital database doesn't reflect the actual number practicing in the hospital and actually they're way less than the available number. All of this contributed to not reaching the goal sample size and had to settle to $78 \%$ response rate.

\section{Declarations}

The authors declare that they have no known competing financial interests or personal relationships that could have appeared to influence the work reported in this paper.

\section{References}


1. Sepsis [Internet]. Who.int. 2021 [cited 29 March 2021]. Available from: https://www.who.int/healthtopics/sepsis\#tab=tab_1

2. Sepsis [Internet]. Who.int. 2021 [cited 29 March 2021]. Available from: https://www.who.int/healthtopics/sepsis\#tab=tab_1

3. Sepsis in Internal Medicine wards: current knowledge, uncertainties and new approaches for management optimization [Internet]. Taylor \& Francis. 2021 [cited 29 March 2021]. Available from: https://www.tandfonline.com/doi/full/10.1080/07853890.2017.1332776

4. Salomão R;Ferreira BL;Salomão MC;Santos SS;Azevedo LCP;Brunialti MKC; Sepsis: evolving concepts and challenges [Internet]. Brazilian journal of medical and biological research $=$ Revista brasileira de pesquisas medicas e biologicas. S. National Library of Medicine; [cited 2021Mar29]. Available from: https://pubmed.ncbi.nlm.nih.gov/30994733/

5. Keeley A, Hine P, Nsutebu E. The recognition and management of sepsis and septic shock: a guide for non-intensivists. 2021.

6. An international sepsis survey: a study of doctors' knowledge and perception about sepsis [Internet]. Rdcu.be. 2021 [cited 29 March 2021]. Available from: https://rdcu.be/chGLm

7. Stamataki P, Papazafiropoulou A, Kalaitzi S, Sarafis P, Kagialari M, Adamou E, et al. Knowledge regarding assessment of sepsis among Greek nurses [Internet]. Journal of infection prevention. SAGE Publications; 2014 [cited 2021Mar29]. Available from: https://www.ncbi.nlm.nih.gov/pmc/articles/PMC5074121/

8. Cowan SL, Holland JA, Frost I, Kane AD. Recognition and management of sepsis by junior doctors [Internet]. Future hospital journal. Royal College of Physicians; 2016 [cited 2021 Mar31]. Available from: https://www.ncbi.nlm.nih.gov/pmc/articles/PMC6465838/

9. Courtney M, Gopinath B, Toward M, Jain R, Rao M. Are adequate fluid challenges prescribed for severe sepsis? [Internet]. International Journal of Health Care Quality Assurance. Emerald Group Publishing Limited; 2014 [cited 2021Mar31]. Available from: https://www.emerald.com/insight/content/doi/10.1108/IJHCQA-01-2014-0013/full/html

10. Roebuck E. Moving sepsis care to the front line: knowledge and views of pre-hospital clinicians [Internet]. Journal of Paramedic Practice. 2015 [cited 2021Mar31]. Available from: https://www.magonlinelibrary.com/doi/abs/10.12968/jpar.2015.7.9.446

11. Mulders MCF, Loots FJ, van Nieuwenhoven J, ter Maaten JC, Bouma HR. Use of sepsis-related diagnostic criteria in primary care: a survey among general practitioners [Internet]. OUP Academic. Oxford University Press; 2021 [cited 2021Mar31]. Available from: https://academic.oup.com/fampra/advance-article/doi/10.1093/fampra/cmab020/6182249? login=true

12. Keeley A, Hine P, Nsutebu E. The recognition and management of sepsis and septic shock: a guide for non-intensivists. Postgraduate Medical Journal. 2017;93(1104):626-634.

13. COVID19 - Global Sepsis Alliance [Internet]. Global Sepsis Alliance. 2021 [cited 31 May 2021]. Available from: https://www.global-sepsis-alliance.org/covid19 
14. The Relationship Between Sepsis and COVID-19: What We Know - bioMérieux Connection [Internet]. bioMérieux Connection. 2021 [cited 31 May 2021]. Available from:

https://www.biomerieuxconnection.com/2020/06/25/the-relationship-between-sepsis-and-covid-19what-we-know/

15. Olwal C, Nganyewo N, Tapela K, Djomkam Zune A, Owoicho O, Bediako Y et al. Parallels in Sepsis and COVID-19 Conditions: Implications for Managing Severe COVID-19. Frontiers in Immunology. $2021 ; 12$.

\section{Figures}

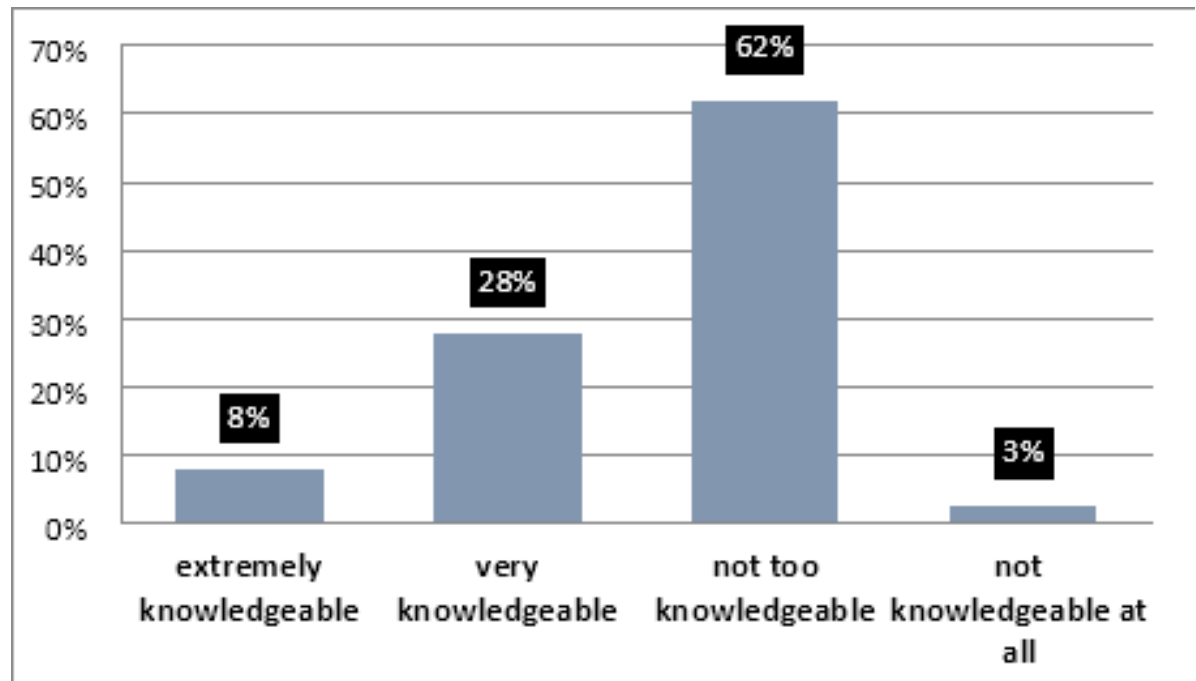

\section{Figure 1}

How knowledgeable are you about sepsis, its treatment and complications associated with it

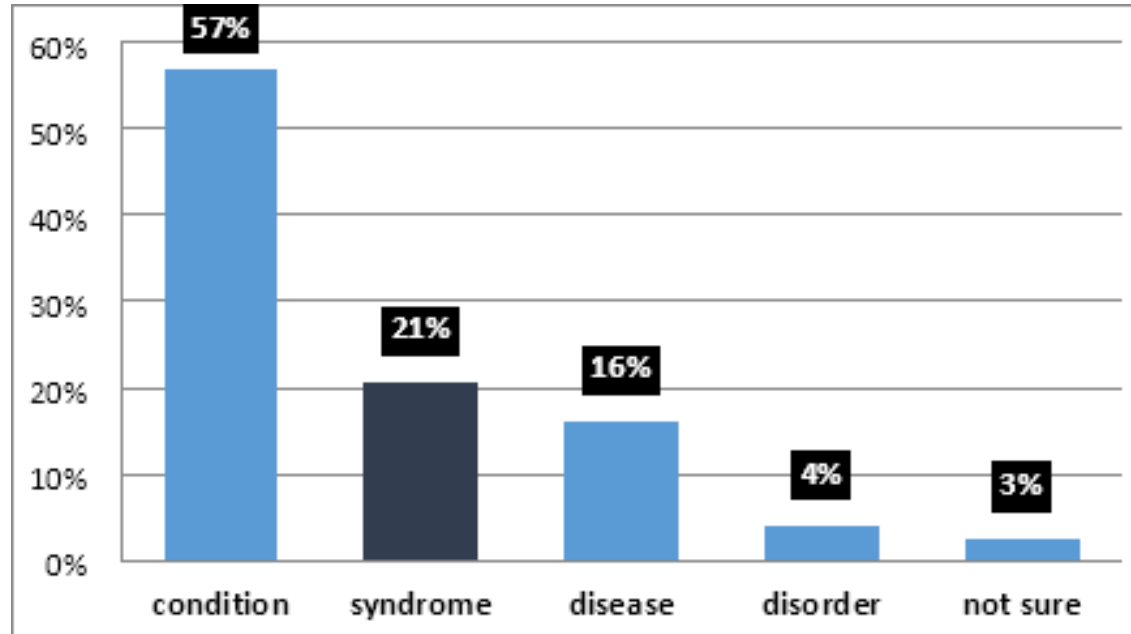

Figure 2

sepsis is... 


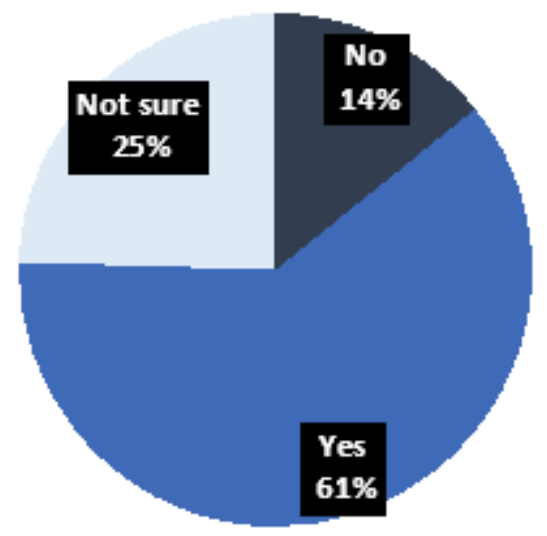

Figure 3

Knowledge about difference between sepsis and septic shock

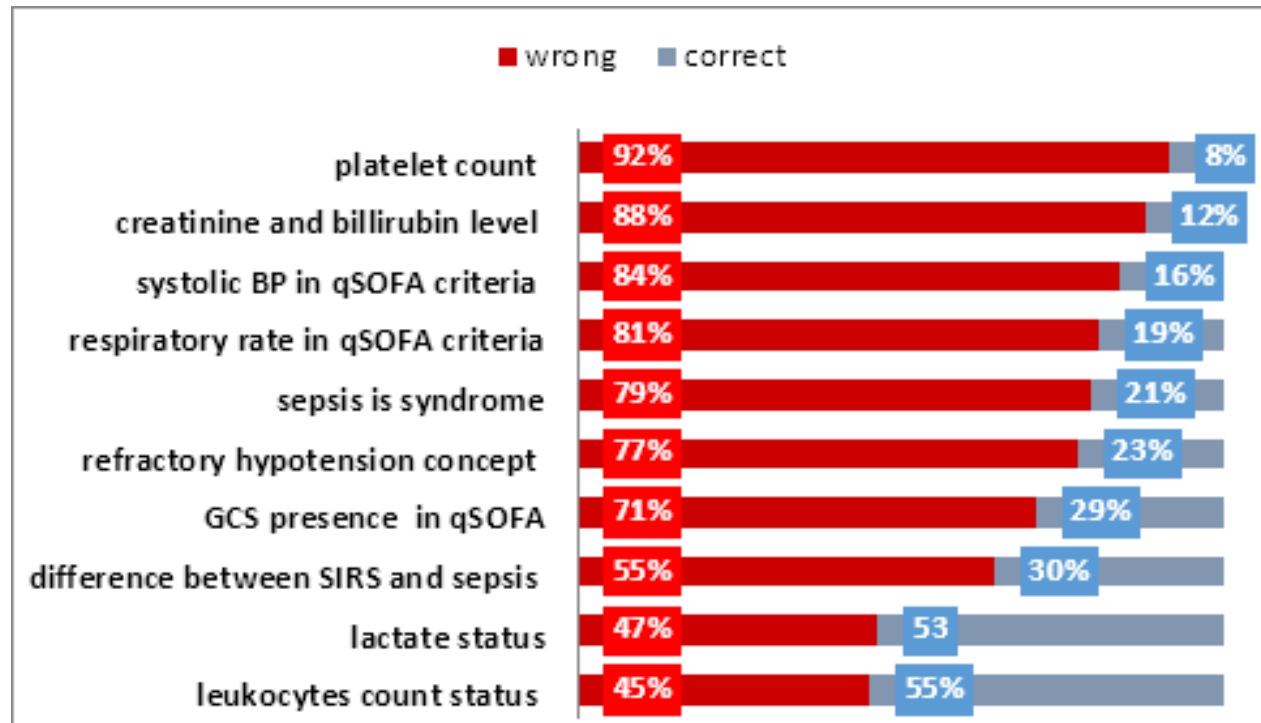

\section{Figure 4}

Participant's response about sepsis diagnosis questions 


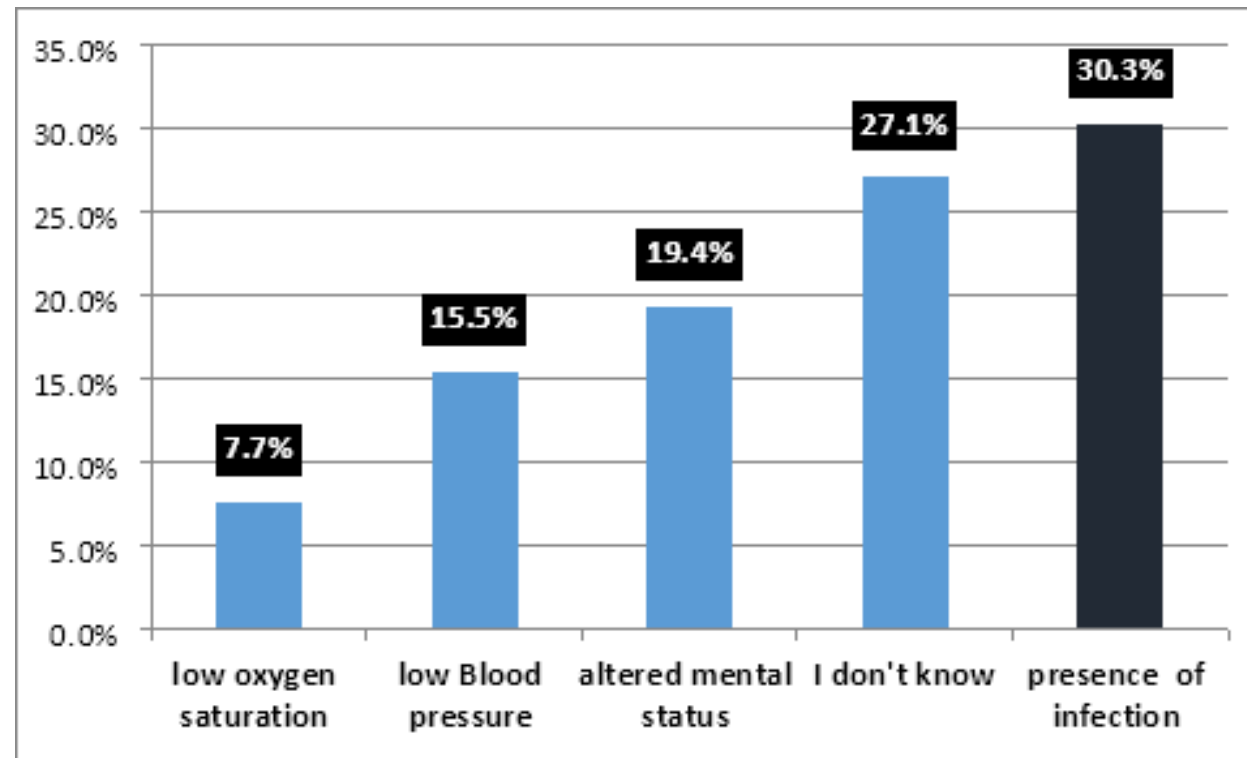

\section{Figure 5}

Knowledge about difference SIRS and sepsis

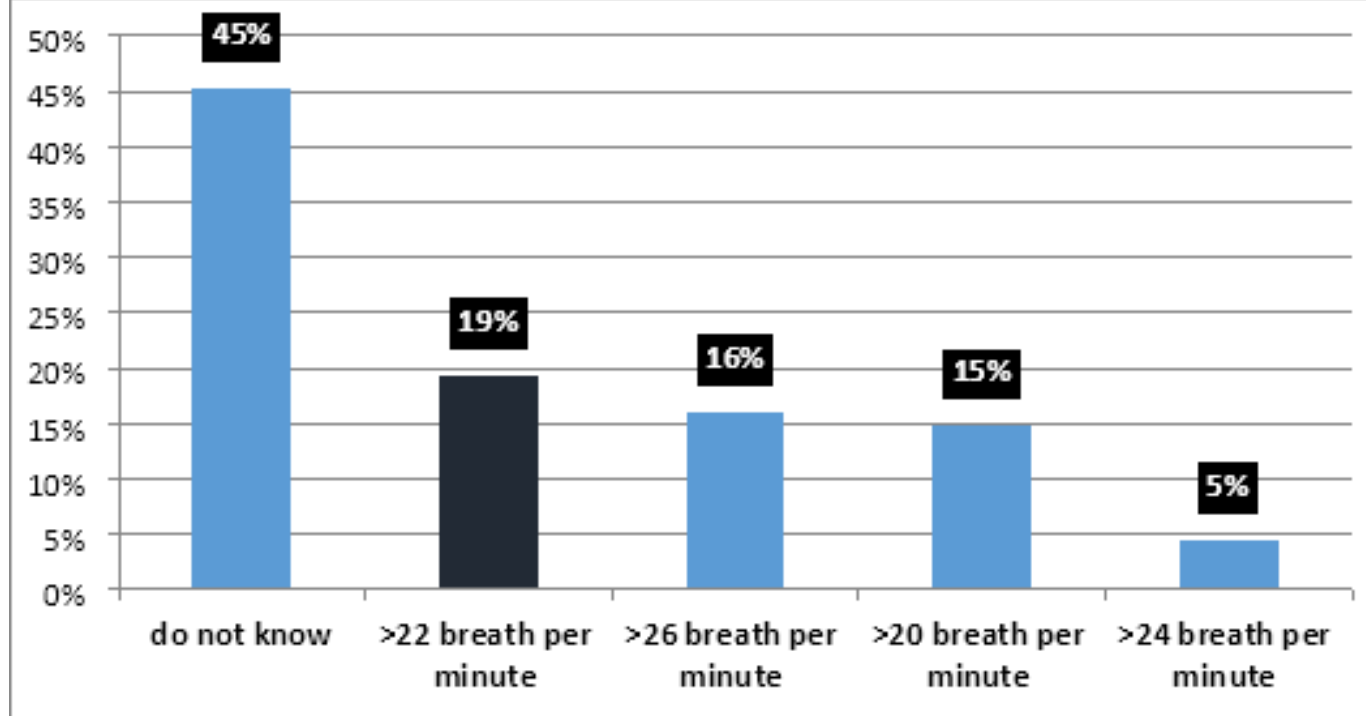

Figure 6

Knowledge about respiratory rate for diagnosis in qSOFA criteria 


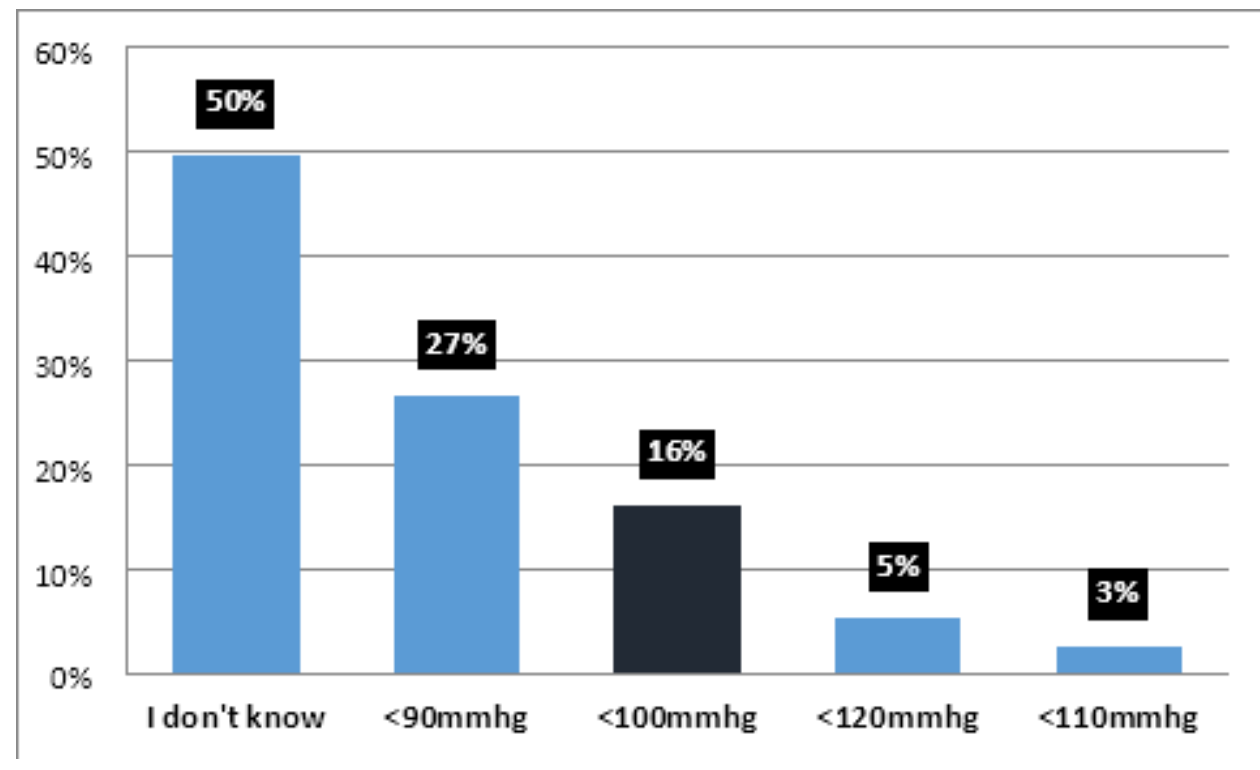

\section{Figure 7}

knowledge about systolic blood pressure for diagnosis in qSOFA criteria

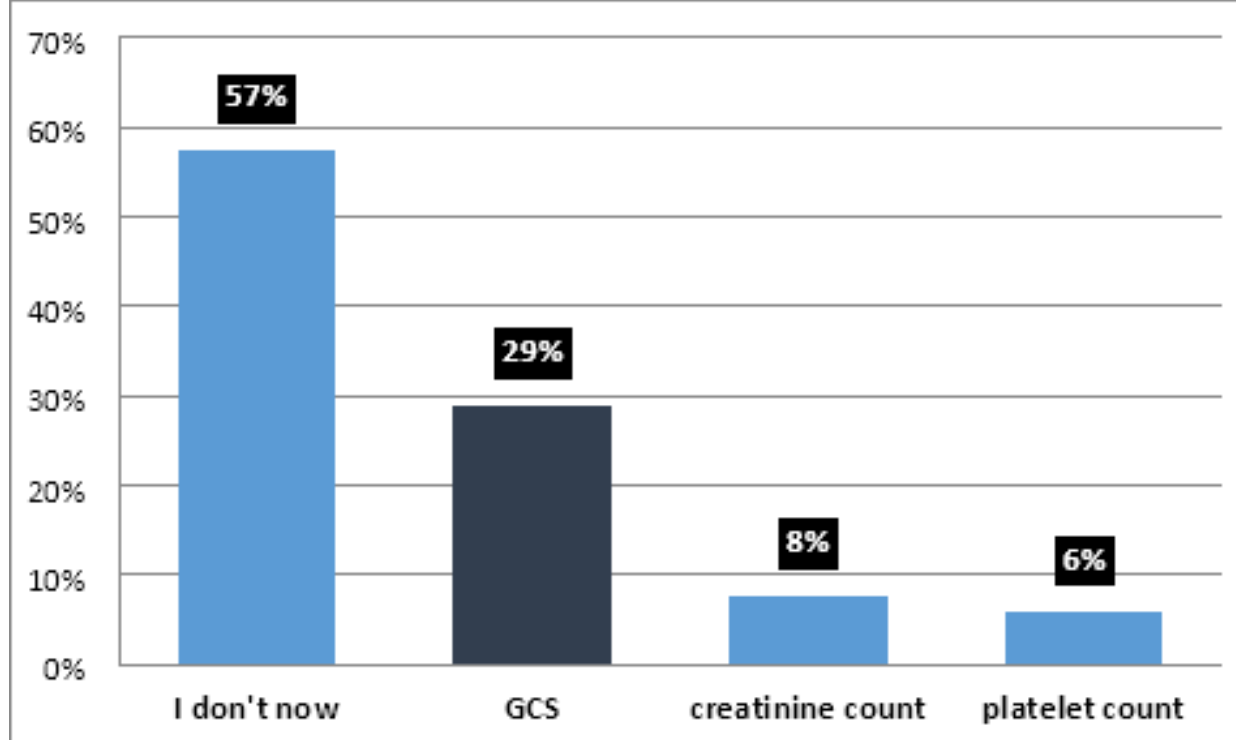

Figure 8

knowledge about feature that include specifically in qSOFA criteria 


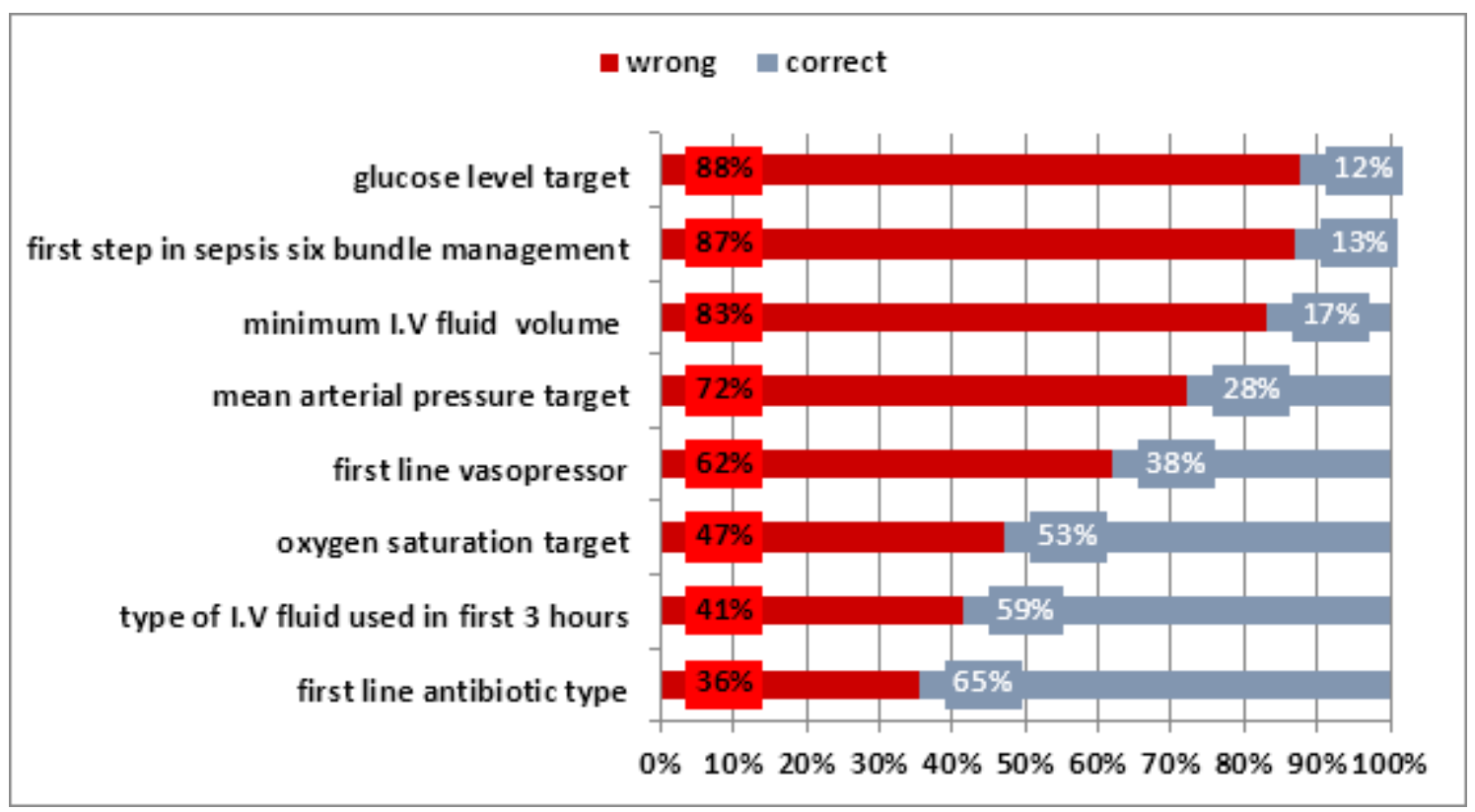

\section{Figure 9}

participant's response about sepsis management questions 